\title{
Crocin inhibits obesity via AMPK-dependent inhibition of adipocyte differentiation and promotion of lipolysis
}

\author{
Ming Gu, Li Luo, Kai Fang* \\ Department of Pharmacy, Union Hospital, Tongji Medical College, Huazhong University of Science and Technology, Wuhan, China.
}

\begin{abstract}
Summary Obesity has become a severe public health problem worldwide. Crocin, a natural product, has been reported to have a number of pharmacological activities, including antiinflammatory, anti-cancer, neuroprotective, antihypertensive, and cardioprotective action. The aims of the current study were to identify the beneficial effects of crocin on obesity, adipocyte differentiation, and lipolysis and to evaluate the possible role of AMPK. Results indicated that crocin significantly increased AMPK phosphorylation in differentiated adipocytes in vitro and in adipose tissue in $\mathrm{db} / \mathrm{db}$ mice. Crocin reduced lipid accumulation in differentiated adipocytes. In addition, crocin inhibited the expression of mRNA of important adipogenesis-related regulators, including CEBP $\alpha$, CEBP $\beta$, PPAR $\gamma$, aP2, FAS, and CD36, in both differentiated adipocytes and adipose tissue in $\mathrm{db} / \mathrm{db}$ mice. Crocin increased the expression of mRNA of key lipolysis-associated factors, including PPAR $\alpha$, LPL, and HSL, in both differentiated adipocytes and adipose tissue in $\mathrm{db} / \mathrm{db}$ mice. In adipocytes, knockdown of AMPK significantly suppressed the crocin-induced inhibition of adipocyte differentiation and increase in lipolysis. BML-275 is an inhibitor of AMPK. In adipose tissue in $\mathrm{db} / \mathrm{db}$ mice, BML-275 suppressed crocin-induced inhibition of fat formation and alleviation of a metabolic disorder. The current results suggest that crocin alleviates obesity in $\mathrm{db} / \mathrm{db}$ mice and that it inhibits adipocyte differentiation in preadipocytes. Crocin inhibits adipogenesis and promotes lipolysis via activation of AMPK. Therefore, crocin may have promise as an option for the clinical treatment for obesity and associated metabolic diseases.
\end{abstract}

Keywords: Obesity, crocin, AMPK, adipocyte differentiation, lipolysis

\section{Introduction}

Obesity and overweight have become a severe public health problem worldwide (1). In the United States, $17.1 \%$ of children and adolescents and $32.2 \%$ of adults were determined to be obese (2). In China, 21 million children were estimated to be overweight, $50 \%$ of which were considered obese (3). Obesity is a strong risk factor for many metabolic diseases, such as type 2 diabetes, and cardiovascular diseases $(4,5)$. Obesity is a state of excessive accumulation of body fat, resulting from increased intake and storage of calories and/or decreased consumption of calories. Fat content is controlled by

*Address correspondence to:

Dr. Kai Fang, Union Hospital, Tongji Medical College, Huazhong University of Science and Technology, No. 1277 Jiefang Avenue, Wuhan 430022, China.

E-mail: xinsilu930@163.com adipocyte differentiation and lipolysis. White adipocytes can store energy and produce numerous hormones and metabolites that modulate glucose and lipid metabolism (6). Increased adipocyte differentiation (7) and enlarged adipocytes are characteristics of obesity (8).

Natural products have long been used in Asia to treat various conditions such as inflammation, obesity, and diabetes (9). Crocin, a carotenoid compound, is a bioactive constituent of both the saffron (Crocus Sativus L.) and gardenia plants (10). Several studies have verified the antioxidant activities of crocin (1114). Crocin has been reported to have a number of pharmacological activities, including anti-inflammatory, anti-cancer, neuroprotective, antihypertensive, and cardioprotective action (15). Studies have reported that crocin lowers blood glucose levels and insulin resistance, providing protection against obesity and improving the lipid profile $(16,17)$. The protective effects of crocin against tissue damage in diabetes have been verified 
(14,17-19). Crocin ameliorated the oxidative burden and inflammatory cascade and it suppressed the progression of diabetic nephropathy in diabetic rats (11). Crocin is effective in relieving the symptoms of obesity and diabetes, including hyperinsulinemia, hyperleptinemia, insulin resistance, and weight gain in animals that were obese or that had type 2 diabetes induced by a high fat diet and streptozotocin (17). In another study in which rats were fed a high-fat diet, crocin was found to alleviate functional and histopathological changes in obese rats in a dose-dependent manner (20). However, the mechanism by which crocin inhibits obesity is not fully understood.

AMP-activated protein kinase (AMPK) is an important sensor and central regulator of metabolism (21). Abnormal regulation of AMPK is associated with obesity, insulin resistance, type 2 diabetes, nonalcoholic fatty liver disease, and cardiovascular disease (21). AMPK has been found to regulate glucose and lipid metabolism by increasing glucose uptake, fatty acid oxidation, mitochondrial biogenesis, and autophagy while suppressing the synthesis of fatty acids, cholesterol, and protein (22).

Preliminary studies by the current authors indicated that crocin upregulated the phosphorylation of AMPK and that it activated AMPK signaling. The aims of the current study were to identify the beneficial effects of crocin on obesity, adipocyte differentiation, and lipolysis and to evaluate the hypothesis that crocin inhibits obesity via activation of AMPK-dependent inhibition of adipogenesis and promotion of lipolysis

\section{Materials and Methods}

\subsection{Cell culture and induction of adipocyte differentiation}

3T3-L1 preadipocytes cells were purchased from the American Type Culture Collection (ATCC; Manassas, VA, USA). 3T3-L1 cells were cultured in DMEM supplemented with heat-inactivated $10 \%$ bovine calf serum (Gibco, CA, USA), $100 \mathrm{mg} / \mathrm{mL}$ penicillin, and $100 \mathrm{mg} / \mathrm{mL}$ streptomycin in a 5\% CO2 incubator at $37^{\circ} \mathrm{C}$.

Induction of adipogenic differentiation was performed as previously reported (23). Two days after reaching confluence (designated day 0 ), pre-adipocytes were cultured in differentiation induction medium (DMI) with DMEM containing 10\% fetal bovine serum (FBS, Gibco, CA, USA), $10 \mu \mathrm{g} / \mathrm{mL}$ insulin (SigmaAldrich, Saint Louis, MO, USA), 0.5 mM 3-isobutyl1-methyl-xanthine (Santa Cruz, USA), and $0.25 \mu \mathrm{M}$ dexamethasone (Sigma-Aldrich, Saint Louis, MO, USA) for 2 days. Next, cells were cultured for an additional 2 days (designated days 2-4) in maturation medium (DMEM containing 10\% FBS and $10 \mu \mathrm{g} / \mathrm{mL}$ insulin) and then maintained in DMEM medium containing 10\%
FBS. The medium was replaced every two days for an additional four days (designated days 4-8) until the cells were harvested. For treatment, $20 \mu \mathrm{M}$ crocin was added to the medium on days $0-8$. The concentration of crocin used was based on previous studies (24).

\subsection{Lentivirus transfection}

Lentivirus (LV)-shAMPK was obtained from Santa Cruz Biotechnology (Santa Cruz, CA, USA). Preadipocytes were transfected with LV-shAMPK according to the manufacture's protocols. 3T3-L1 preadipocytes with stable knockdown of AMPK were produced.

\subsection{Lipid accumulation determination}

After the induction of adipogenic differentiation, cells were washed with phosphate-buffered saline (PBS), fixed with $10 \%$ formaldehyde for $30 \mathrm{~min}$, and then washed with distilled water. The cells were then stained with BODIPY and Hoechst reagent for $20 \mathrm{~min}$ at room temperature. Cells were then washed with PBS and viewed under a confocal microscope (Olympus, Japan).

\subsection{Animal experiments}

All animal experiments were approved by the Institutional Animal Care and Use Committee of Union Hospital, Tongji Medical College, Huazhong University of Science and Technology and were conducted in accordance with ARRIVE and NIH guidelines for animal welfare. C57BL/KsJ-Lepdb (db/db) mice and their lean littermates (wild-type) were obtained from the Model Animal Research Center of Nanjing University. Ten-week-old male mice were housed at $22 \pm 2{ }^{\circ} \mathrm{C}$ and $60 \pm 5 \%$ relative humidity and kept on a 12-h light/dark cycle with free access to water and a regular chow diet. $\mathrm{db} / \mathrm{db}$ mice were randomly divided into three groups (db/db, Crocin, and Crocin+BML-275, $n=10$ in each group). Mice in the Crocin+BML-275 group were intraperitoneally injected with BML-275 before the administration of crocin. Mice in the Crocin group were given $20 \mathrm{mg} / \mathrm{kg}$ of crocin daily. The dose of crocin was selected based on a previous study (25). Wild-type (WT) mice were treated with the vehicle in the same manner as the normal controls. This experiment lasted 8 weeks. Blood samples were collected, and tissue samples were flash-frozen in liquid nitrogen or collected for embedding in paraffin.

\subsection{Histological examination of adipose tissue}

A histological examination was performed to evaluate morphological changes in adipose tissue. Paraffinembedded adipose tissue sections $(5 \mu \mathrm{m})$ were prepared and stained with H\&E. Images were captured using a light microscope (Olympus, Japan). 


\subsection{Metabolic and biochemical analysis}

Before the OGTT and IPITT, mice were fasted for 12 $\mathrm{h}$ and $6 \mathrm{~h}$, respectively. Blood glucose was measured $0,30,60,90$, and $120 \mathrm{~min}$ after the administration of glucose and insulin. Blood glucose levels were determined using tail vein blood via the Accu-Chek glucometer (Roche, Basel, Switzerland). At the end of the experiment, mice were fasted for $12 \mathrm{~h}$ and blood samples were collected from the orbital venous plexus immediately prior to sacrifice. Serum levels of triglycerides (TG), non-esterified fatty acids (NEFA), and total cholesterol (TC) were quantified using commercial kits (Nanjing Jiancheng, Nanjing, China). Serum insulin levels were determined using an enzymelinked-immunosorbent assay (ELISA) kit (Invitrogen, Carlsbad, CA, USA).

\subsection{Real-time quantitative $P C R$}

Total RNA from liver tissue was isolated using TRIzol reagent (Life Technologies, Carlsbad, CA, USA). RNA samples were treated with DNase and reversetranscribed into cDNA using Superscript II (Life Technologies, Foster City, CA). Real-time RT-PCR was performed with SYBR Green PCR Master Mix (Takara, Tokyo, Japan) in an ABI StepOnePlus Real-time PCR System. The expression of mRNA for each target gene was normalized relative to that of glyceraldehyde 3-phosphate dehydrogenase (GAPDH).

\subsection{Western blot analysis}

Liver tissues were lysed in RIPA Lysis Buffer (Beyotime, Jiangsu, China). After determination of the protein concentration using the BCA Protein Assay Kit (Beyotime, Jiangsu, China), the samples were separated with sodium dodecyl sulphate-polyacrylamide gel electrophoresis (SDS-PAGE) and transblotted onto polyvinylidene fluoride membranes (PVDF, Millipore). Membranes were then incubated with primary antibodies (diluted 1:1000; Cell Signaling Technology, USA) overnight at $4{ }^{\circ} \mathrm{C}$. Membranes were washed with TBST and then were incubated with horseradish-peroxidase (HRP)-conjugated secondary antibody (diluted 1:5,000; Thermo Fisher Scientific, USA) for $1 \mathrm{~h}$ at room temperature. Finally, the blots were visualized using chemiluminescence (ECL) detection reagents (Thermo Fisher Scientific, USA). Data were analyzed by calculating the integrated optical density.

\subsection{Statistical analysis}

Results are expressed as the mean \pm SD. Significant differences among groups were assessed using oneway ANOVA followed by Dunnett's test. Statistical significance was defined as $p<0.05$.

\section{Results} 3.1. Crocin upregulates AMPK phosphorylation and
lipid accumulation in differentiated adipocytes

A 3T3-L1 adipogenic differentiation model was used to evaluate the effect of crocin on the course of adipocyte differentiation. Phosphorylation was inhibited with LV-shAMPK in differentiated adipocytes, but crocin significantly increased that phosphorylation, as shown in Figure 1A. Figure 1B shows that crocin markedly increased BODIPY staining in differentiated adipocytes, indicating a reduction in lipid accumulation and inhibition of adipocyte differentiation. In addition, knockdown of AMPK significantly blocked crocin's action on lipid accumulation in differentiated adipocytes (Figure 1B). Findings indicated that crocin inhibited adipocyte differentiation via upregulation of AMPK signaling.

\subsection{Crocin inhibits adipogenic differentiation and promotes lipolysis in differentiated adipocytes}

To investigate the mechanism of crocin-induced inhibition of lipid accumulation in differentiated adipocytes, the expression of adipogenesis-related factors and lipolysis-associated regulators was determined. As shown in Figure 2A-F, crocin reduced the expression of mRNA of important adipogenesis-related regulators, including CCAAT/enhancer binding protein $\alpha(\mathrm{CEBP} \alpha)$, CEBP $\beta$, PPAR $\gamma$, aP2, FAS, and CD36, in differentiated adipocytes. Moreover, treatment with crocin increased the expression of key lipolysis-associated regulators such as PPAR $\alpha$, lipoprotein lipase (LPL), and hormonesensitive lipase (HSL) (Figure 2G-I). Results indicated that the inhibition of lipid accumulation by crocin in differentiated adipocytes may be attributed to its inhibition of adipogenic differentiation and its promotion of lipolysis. Moreover, these actions of crocin were inhibited by the knockdown of AMPK (Figures 2AI). These findings suggest that treatment with crocin resulted in a reduction in adipocyte differentiation and an increase in lipolysis in an AMPK-dependent manner.

\subsection{Crocin inhibits adipose formation in diabetic and obese $\mathrm{db} / \mathrm{db}$ mice}

To evaluate the effect of crocin on adipose formation in vivo, $\mathrm{db} / \mathrm{db}$ mice were injected with crocin. Figure $3 \mathrm{~A}$ and $\mathrm{B}$ show that AMPK phosphorylation in adipose tissue was significantly lower than that in WT mice. Treatment with crocin significantly increased AMPK phosphorylation in adipose tissue in $\mathrm{db} / \mathrm{db}$ mice (Figure $3 \mathrm{~A}$ and B). BML-275 decreased AMPK phosphorylation in adipose tissue in the presence of crocin (Figure $3 \mathrm{~A}$ and $\mathrm{B})$. Findings indicated that crocin decreased the weight of epidydimal and perirenal white adipose 


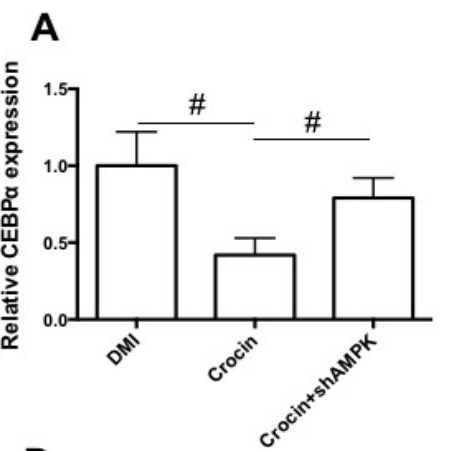

B
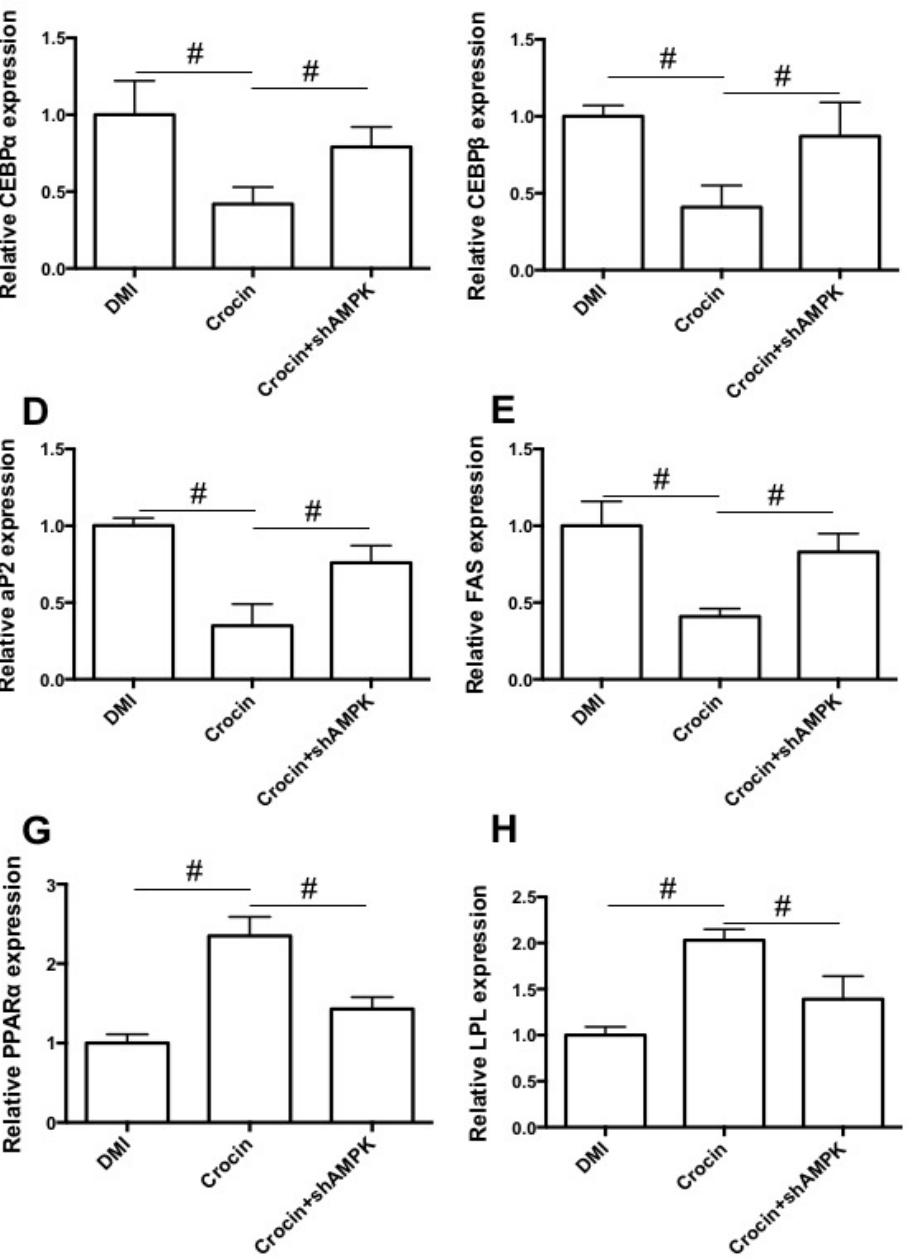

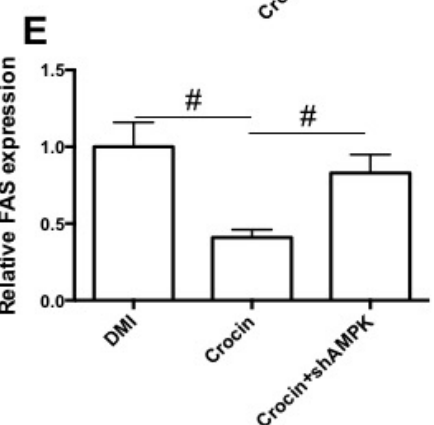

H

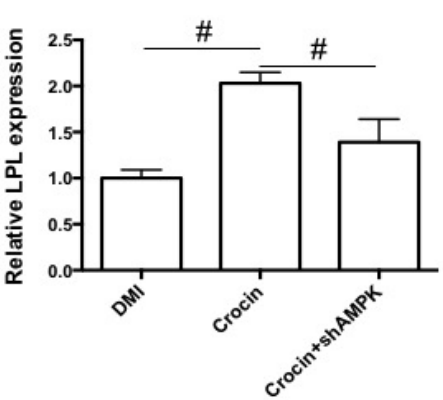

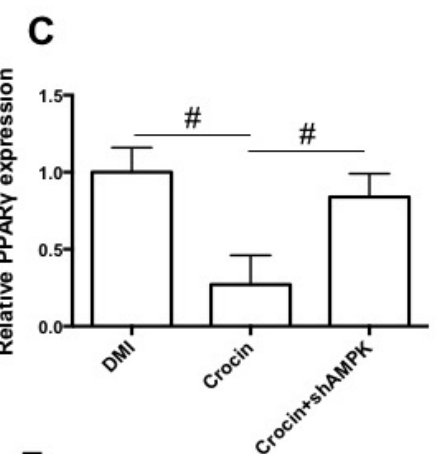

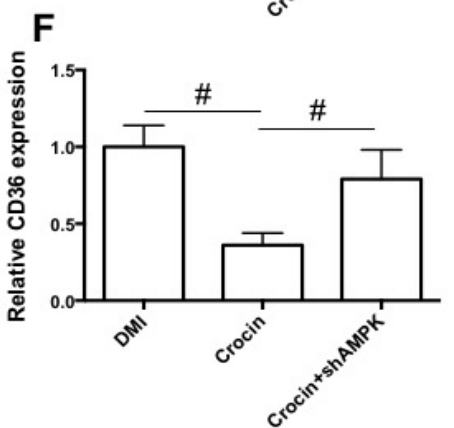

I

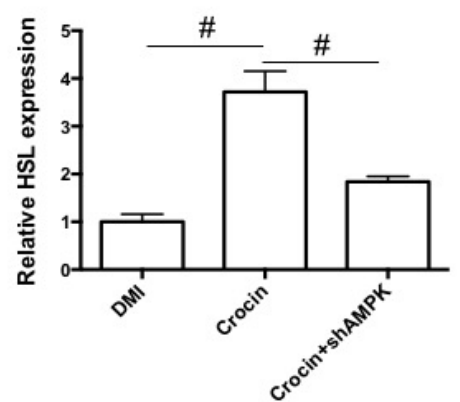

Figure 2. Effects of crocin on the expression of regulators of adipogenesis and lipolysis in differentiated adipocytes. Expression of CEBP $\alpha$ (A), CEBP $\beta$ (B), PPAR $\gamma$ (C), aP2 (D), FAS (E), CD36 (F), PPAR $\alpha$ (G), LPL (H), and HSL (I) mRNA in differentiated adipocytes was determined using real-time qPCR. ${ }^{\#} p<0.05$ indicates a significant difference between the two groups.
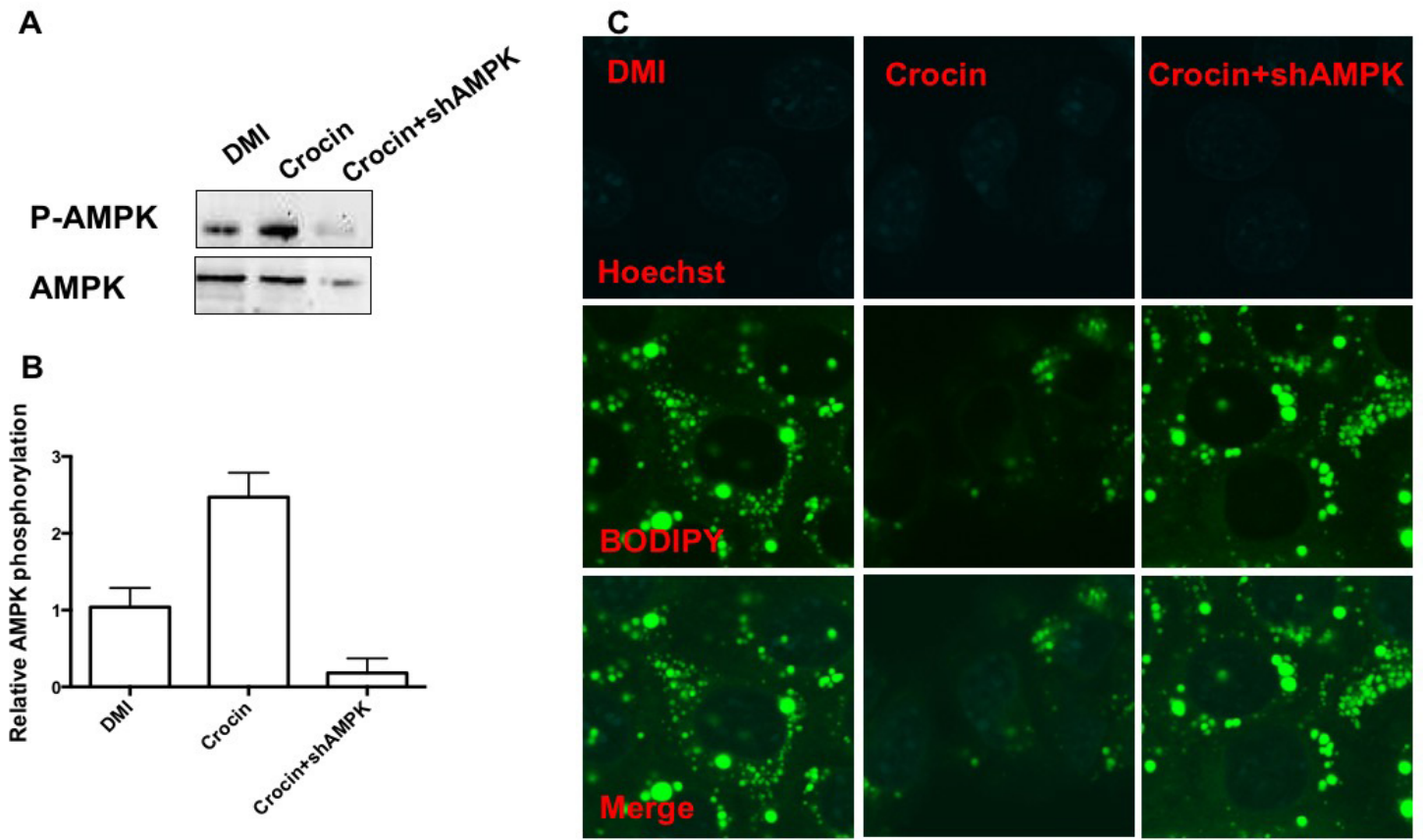

Figure 1. Effects of crocin on AMPK phosphorylation and lipid accumulation in differentiated adipocytes. (A) Western blot analysis of AMPK phosphorylation in differentiated adipocytes. (B) Statistical analysis of AMPK phosphorylation. (C) BODIPY staining of differentiated adipocytes. ${ }^{\#} p<0.05$ indicates a significant difference between the two groups. 
A

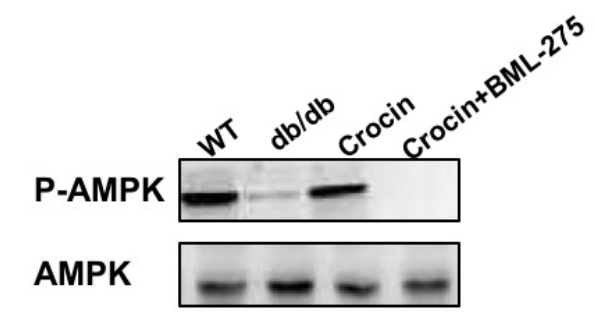

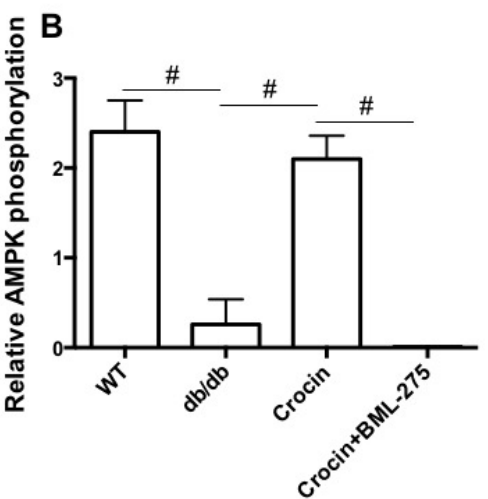

C
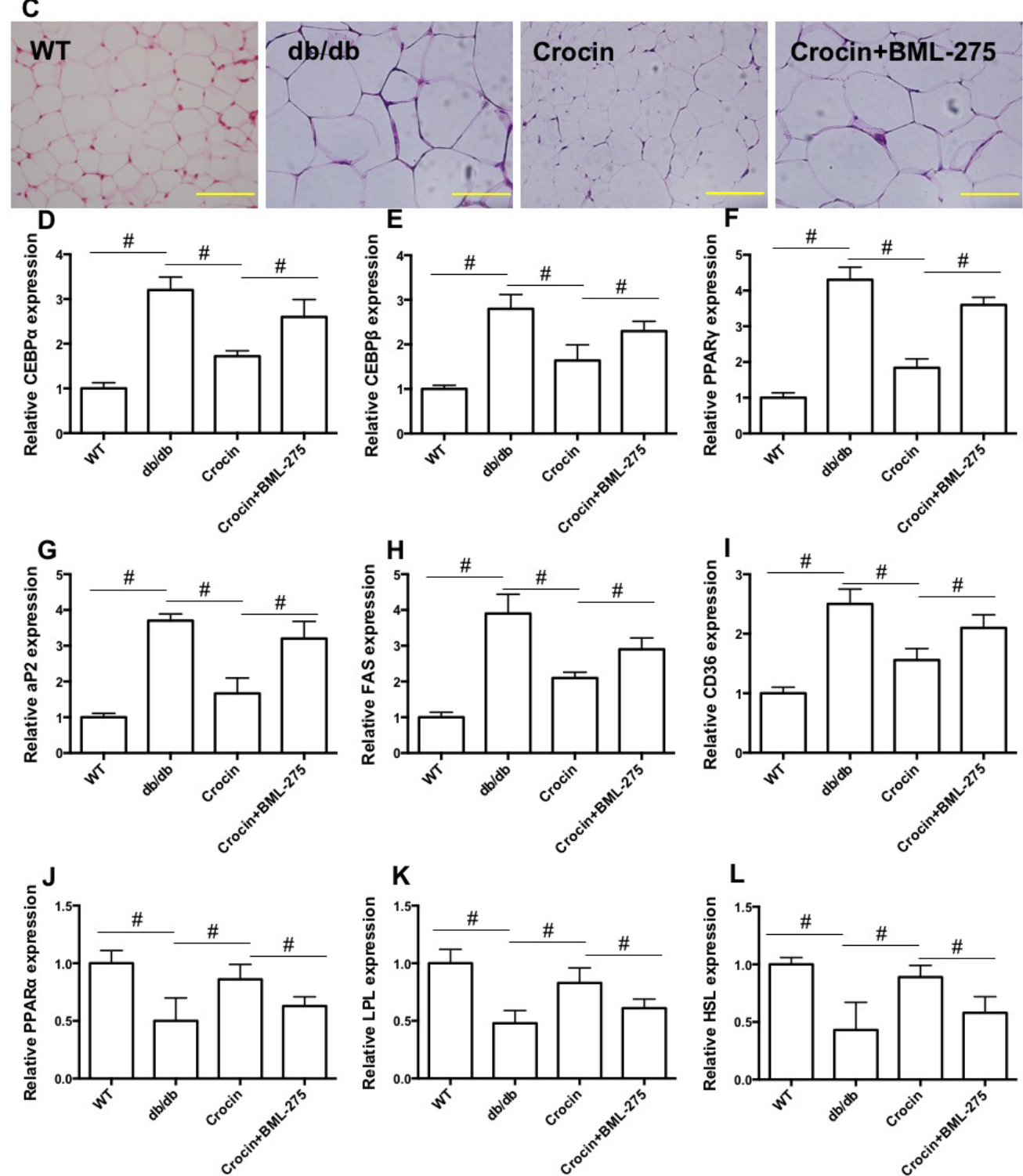

L

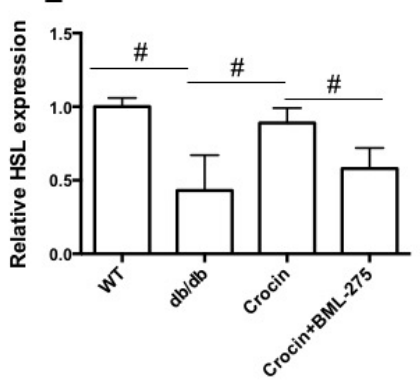

Figure 3. Effects of crocin on AMPK phosphorylation, adipocyte size, and the expression of regulators of adipogenesis and lipolysis in adipose tissue. (A) Western blot analysis of AMPK phosphorylation in differentiated adipocytes. (B) statistical analysis of AMPK phosphorylation. (C) HE staining of adipose tissue. Expression of CEBP $\alpha$ (D), CEBP $\beta$ (E), PPAR $\gamma$ (F), aP2 $(\mathbf{G})$, FAS (H), CD36 (I), PPAR $\alpha(\mathbf{J})$, LPL (K), and HSL (L) mRNA in differentiated adipocytes was determined using real-time qPCR. ${ }^{\#} p<0.05$ indicates a significant difference between the two groups.

tissue, though this action was inhibited by injection of BML-275, an inhibitor of AMPK (Table 1). Figure 3C shows that crocin decreased the size of adipocytes in $\mathrm{db} / \mathrm{db}$ mice. However, injection of BML-275 reversed the crocin-induced shrinkage in adipocyte size in $\mathrm{db} /$ $\mathrm{db}$ mice (Figure 3C). Moreover, crocin reduced the expression of mRNA of important adipogenesisrelated regulators, including $\mathrm{CEBP} \alpha, \mathrm{CEBP} \beta, \operatorname{PPAR} \gamma$, 
Table 1. Effects of crocin on general parameters in $\mathrm{db} / \mathrm{db}$ mice

\begin{tabular}{lcccc}
\hline Parameters & \multicolumn{1}{c}{ WT } & $\mathrm{db} / \mathrm{db}$ & Crocin & Crocin+BML-275 \\
\hline Body weight (g) & $25.4 \pm 1.7$ & $43.7 \pm 2.5^{\#}$ & $34.3 \pm 2.3^{\# \#}$ & $39.7 \pm 2.1^{\# \#}$ \\
Epididymal adipose weight $(\mathrm{g})$ & $0.19 \pm 0.07$ & $2.53 \pm 0.23^{\#}$ & $1.91 \pm 0.16^{\# \#}$ & $2.34 \pm 0.12^{\# \#}$ \\
Perirenal adipose weight $(\mathrm{g})$ & $0.1 \pm 0.04$ & $1.35 \pm 0.08^{\#}$ & $0.98 \pm 0.13^{\# \#}$ & $1.22 \pm 0.11^{\# \# \#}$ \\
Serum NEFA (mM) & $0.22 \pm 0.05$ & $0.71 \pm 0.12^{\#}$ & $0.45 \pm 0.11^{\# \#}$ & $0.62 \pm 0.08^{\# \#}$ \\
Serum TG (mM) & $0.94 \pm 0.09$ & $1.53 \pm 0.16^{\#}$ & $1.14 \pm 0.13^{\# \#}$ & $1.38 \pm 0.08^{\# \#}$ \\
Serum TC (mM) & $2.02 \pm 0.16$ & $2.89 \pm 0.20^{\#}$ & $2.43 \pm 0.11^{\# \#}$ & $2.72 \pm 0.12^{\# \# \#}$ \\
Blood glucose (mM) & $5.97 \pm 0.82$ & $16.4 \pm 2.81^{\#}$ & $9.6 \pm 1.15^{\# \#}$ & $14.5 \pm 2.15^{\# \# \#}$ \\
Serum insulin $(\mathrm{ng} / \mathrm{mL})$ & $2.8 \pm 0.43$ & $18.7 \pm 1.18^{\#}$ & $10.9 \pm 3.11^{\# \#}$ & $16.3 \pm 2.25^{\# \# \#}$ \\
\hline
\end{tabular}

WT, wild type; $\mathrm{db} / \mathrm{db}, \mathrm{db} / \mathrm{db}$ mice; \#, $p<0.05$, compared with WT; \#, $p<0.05$, compared with $\mathrm{db} / \mathrm{db}$ mice; \#\#, $p<0.05$, compared with Crocin treatment.

A

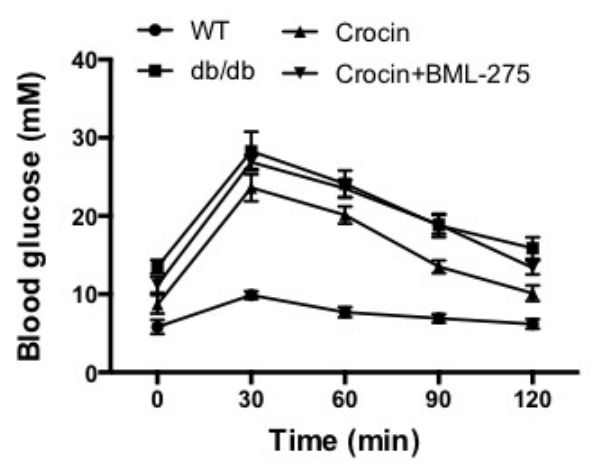

C

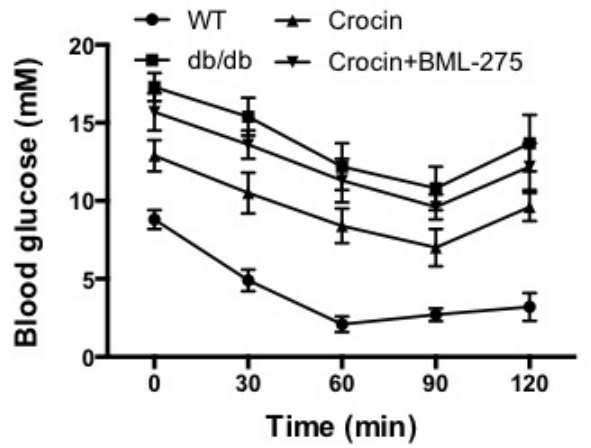

B
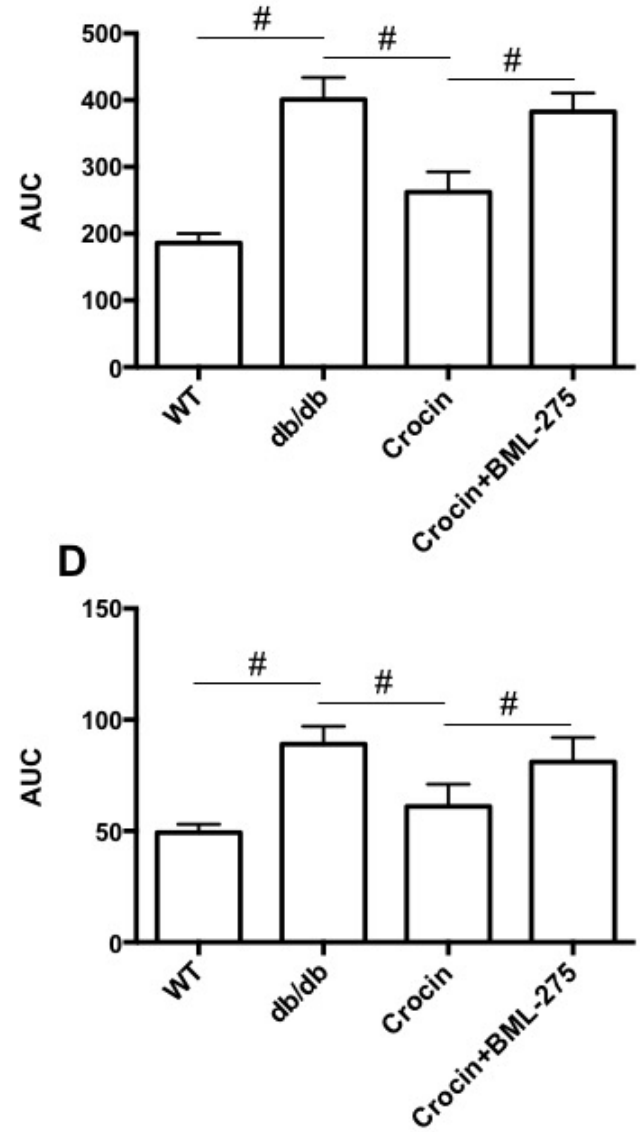

Figure 4. Effects of crocin on general glucose and lipid metabolism in $\mathbf{~ d b / d b ~ m i c e . ~ ( A ) ~ O G T T ~ t e s t . ~ ( B ) ~ a r e a ~ u n d e r ~ t h e ~ c u r v e ~}$ during the OGTT. (C) IPITT test. (D) area under the curve during the IPITT. ${ }^{*} p<0.05$ indicates a significant difference between the two groups.

aP2, FAS, and CD36, in adipose tissue (Figure 3DI). Treatment with crocin increased the expression of key lipolysis-associated regulators such as PPAR $\alpha$, LPL, and HSL in adipose tissue (Figure 3J-L). Crocin's action on adipocyte differentiation and lipolysis was inhibited by BML-275 (Figure 3D-L). Results indicated that crocin suppressed the formation of adipose by inhibiting adipogenesis and promoting lipolysis via AMPK-dependent regulation of key regulators.

3.4. Crocin attenuates a metabolic disorder in diabetic and obese $\mathrm{db} / \mathrm{db}$ mice
The protective effect of crocin against systemic metabolic disorder was evaluated in $\mathrm{db} / \mathrm{db}$ mice. As shown in Table 1, crocin reduced body weight, fasting blood glucose levels, and serum levels of insulin, TG, $\mathrm{TC}$, and NEFA in $\mathrm{db} / \mathrm{db}$ mice, but these effects of crocin were inhibited by BML-275. In addition, treatment with crocin reduced glucose levels at different times after the administration of glucose and insulin (Figure 4A-D). Improvement in glucose and insulin tolerance as a result of crocin was inhibited by BML-275. Findings suggested that crocin provided protection against a systemic metabolic disorder via upregulation of AMPK signaling. 


\section{Discussion}

Obesity is a result of excessive accumulation of fat in the body (26), and dysregulated adipogenesis and lipolysis are involved in this process. Adipogenic differentiation and maturation of pre-adipocytes are complex processes, and a battery of regulators are involved in the regulation of the formation of adipose. In response to adipogenic stimuli, expression of CEBP $\beta$ initiates the differentiation process (27), which is followed by the sequential activation of various key regulators such as CEBP $\alpha$ and PPAR $\gamma$ (28). During differentiation, FAS and other fat synthesis-related genes are activated, leading to the synthesis of lipids droplets and storage of TG (29). Lipolysis, the catabolic pathway of the fatty acid cycle, is also crucial to balanced fat metabolism. Enhanced lipolysis contributes to the release of fatty acids and a decrease in fat deposition. The lipolytic process is mainly controlled by PPAR $\alpha$, LPL, HSL, and other key regulators. LPL is the rate-limiting enzyme for the hydrolysis of TG and very low-density lipoproteins (30).

The current study evaluated the effect of crocin on obesity with a focus on adipocyte differentiation and lipolysis in 3T3-L1 cells. Findings revealed that administration of crocin significantly reduced body weight and fat mass in obese mice, suggesting that crocin may be a potential option as an intervention to fight obesity and associated metabolic disorders. In addition, results indicated that crocin significantly reduced lipid accumulation in differentiated adipocytes. Moreover, crocin decreased the weight of adipose tissue in $\mathrm{db} / \mathrm{db}$ mice, helping to alleviate a systemic metabolic disorder in mice. The possible mechanisms of these actions were examined, and crocin was found to inhibit the expression of adipogenesis-related factors and to promote the expression of lipolysis-associated regulators. The findings support the hypothesis that crocin inhibits the formation of adipose tissue and thus protects against systemic metabolic dysfunction.

AMPK is a serine/threonine protein that is an important regulatory sensor of cellular energy metabolism (31). Activated AMPK inhibits acetyl-CoA carboxylase (ACC), which is an essential enzyme for lipid biosynthesis (32). During adipogenesis, AMPK inhibits the expression of sterol regulatory element binding protein-1 (SREBP-1), CEBP $\alpha, \operatorname{PPAR} \gamma$, and FAS (33), resulting in suppression of the differentiation of adipocytes. In addition, AMPK has been found to promote lipolysis and thus decrease the mass of adipose tissue (34). Preliminary studies by the current authors indicated that AMPK was activated by crocin. The current study examined whether activation of AMPK was involved in crocin's protection against obesity. Results revealed that downregulation of AMPK via lentivirus transfection inhibited crocin-induced suppression of lipid accumulation, downregulation of the expression of adipogenesis-related regulators, and upregulation of the expression of lipolysis-associated factors in vitro. BML-275 is an inhibitor of AMPK. In vivo, BML-275 reversed crocin's inhibition of obesity and diabetes. Results indicated that activation of AMPK was involved in crocin's protection from obesity and diabetes via regulation of adipocyte differentiation and lipolysis.

The current results suggest that crocin alleviated obesity in $\mathrm{db} / \mathrm{db}$ mice and that it inhibited adipocyte differentiation in preadipocytes. Crocin inhibits adipogenesis and it promotes lipolysis via activation of AMPK. Therefore, crocin may have promise as an option for the clinical treatment for obesity and associated metabolic diseases. Further studies are needed to verify crocin's potential in other animal models and to explore the specific and non-specific biological actions of crocin.

\section{References}

1. Flier JS. Obesity wars: Molecular progress confronts an expanding epidemic. Cell. 2004; 116:337-350.

2. Ogden CL, Carroll MD, Curtin LR, McDowell MA, Tabak CJ, Flegal KM. Prevalence of overweight and obesity in the United States, 1999-2004. JAMA. 2006; 295:1549-1555.

3. Wise J. Obesity rates rise substantially worldwide. BMJ (Clinical research ed). 2014; 348:g3582.

4. Matsuda M, Shimomura I. Increased oxidative stress in obesity: Implications for metabolic syndrome, diabetes, hypertension, dyslipidemia, atherosclerosis, and cancer. Obes Res Clin Pract. 2013; 7:e330-341.

5. Haslam DW, James WP. Obesity. Lancet (London, England). 2005; 366:1197-1209.

6. Choe SS, Huh JY, Hwang IJ, Kim JI, Kim JB. Adipose tissue remodeling: Its role in energy metabolism and metabolic disorders. Front Endocrinol (Lausanne). 2016; $7: 30$

7. Camp HS, Ren D, Leff T. Adipogenesis and fat-cell function in obesity and diabetes. Trends Mol Med. 2002; 8:442-447.

8. Muir LA, Neeley CK, Meyer KA, et al. Adipose tissue fibrosis, hypertrophy, and hyperplasia: Correlations with diabetes in human obesity. Obesity (Silver Spring, Md). 2016; 24:597-605.

9. Vasudeva N, Yadav N, Sharma SK. Natural products: A safest approach for obesity. Chin J Integr Med. 2012; 18:473-480.

10. Esposito E, Drechsler M, Mariani P, Panico AM, Cardile V, Crasci L, Carducci F, Graziano ACE, Cortesi R, Puglia C. Nanostructured lipid dispersions for topical administration of crocin, a potent antioxidant from saffron (Crocus sativus L.). Mater Sci Eng C Mater Biol Appl. 2017; 71:669-677.

11. Abou-Hany HO, Atef H, Said E, Elkashef HA, Salem HA. Crocin mediated amelioration of oxidative burden and inflammatory cascade suppresses diabetic nephropathy progression in diabetic rats. Chem Biol Interact. 2018; 284:90-100.

12. Li S, Liu X, Lei J, Yang J, Tian P, Gao Y. Crocin protects podocytes against oxidative stress and inflammation induced by high glucose through inhibition of NF- 
kappaB. Cell Physiol Biochem. 2017; 42:1481-1492.

13. Yang X, Huo F, Liu B, Liu J, Chen T, Li J, Zhu Z, Lv B. Crocin inhibits oxidative stress and pro-inflammatory response of microglial cells associated with diabetic retinopathy through the activation of PI3K/Akt signaling pathway. J Mol Neurosci. 2017; 61:581-589.

14. Yaribeygi H, Mohammadi MT, Sahebkar A. Crocin potentiates antioxidant defense system and improves oxidative damage in liver tissue in diabetic rats. Biomed Pharmacother. 2018; 98:333-337.

15. Yosri H, Elkashef WF, Said E, Gameil NM. Crocin modulates IL-4/IL-13 signaling and ameliorates experimentally induced allergic airway asthma in a murine model. Int Immunopharmacol. 2017; 50:305-312.

16. Mashmoul M, Azlan A, Khaza'ai H, Yusof BN, Noor SM. Saffron: A natural potent antioxidant as a promising antiobesity drug. Antioxidants (Basel, Switzerland). 2013; 2:293-308.

17. Hazman O, Aksoy L, Buyukben A. Effects of crocin on experimental obesity and type- 2 diabetes. Turk J Med Sci. 2016; 46:1593-1602.

18. Altinoz E, Oner Z, Elbe H, Cigremis Y, Turkoz Y. Protective effects of saffron (its active constituent, crocin) on nephropathy in streptozotocin-induced diabetic rats. Hum Exp Toxicol. 2015; 34:127-134.

19. Sepahi S, Mohajeri SA, Hosseini SM, Khodaverdi E, Shoeibi N, Namdari M, Tabassi SAS. Effects of crocin on diabetic maculopathy: A placebo-controlled randomized clinical trial. Am J Ophthalmol. 2018; 190:89-98.

20. Mashmoul M, Azlan A, Mohtarrudin N, Mohd Yusof BN, Khaza'ai H, Khoo HE, Farzadnia M, Boroushaki MT. Protective effects of saffron extract and crocin supplementation on fatty liver tissue of high-fat dietinduced obese rats. BMC Complement Altern Med. 2016; 16:401.

21. Day EA, Ford RJ, Steinberg GR. AMPK as a therapeutic target for treating metabolic diseases. Trends Endocrinol Metab. 2017; 28:545-560.

22. Hardie DG, Ross FA, Hawley SA. AMPK: A nutrient and energy sensor that maintains energy homeostasis. Nat Rev Mol Cell Biol. 2012; 13:251-262.

23. Choi RY, Lee HI, Ham JR, Yee ST, Kang KY, Lee MK. Heshouwu (Polygonum multiflorum Thunb.) ethanol extract suppresses pre-adipocytes differentiation in 3T3-L1 cells and adiposity in obese mice. Biomed Pharmacother. 2018; 106:355-362.

24. Mousavi SH, Tayarani NZ, Parsaee H. Protective effect of saffron extract and crocin on reactive oxygen speciesmediated high glucose-induced toxicity in PC12 cells. Cell Mol Neurobiol. 2010; 30:185-191.

25. Feidantsis K, Mellidis K, Galatou E, Sinakos Z, Lazou A. Treatment with crocin improves cardiac dysfunction by normalizing autophagy and inhibiting apoptosis in STZinduced diabetic cardiomyopathy. Nutr Metab Cardiovasc Dis. 2018; 28:952-961.

26. Zhang Y, Liu J, Yao J, Ji G, Qian L, Wang J, Zhang G, Tian J, Nie Y, Zhang YE, Gold MS, Liu Y. Obesity: Pathophysiology and intervention. Nutrients. 2014; 6:5153-5183.

27. Gregoire FM, Smas CM, Sul HS. Understanding adipocyte differentiation. Physiol Rev. 1998; 78:783-809.

28. Tontonoz P, Hu E, Spiegelman BM. Stimulation of adipogenesis in fibroblasts by PPAR gamma 2, a lipidactivated transcription factor. Cell. 1994; 79:1147-1156.

29. Gregoire FM. Adipocyte differentiation: From fibroblast to endocrine cell. Exp Biol Med (Maywood). 2001; 226:997-1002.

30. Kersten S. Physiological regulation of lipoprotein lipase. Biochim Biophys Acta. 2014; 1841:919-933.

31. Kim SK, Kong CS. Anti-adipogenic effect of dioxinodehydroeckol via AMPK activation in 3T3-L1 adipocytes. Chem Biol Interact. 2010; 186:24-29.

32. Xi Y, Wu M, Li H, Dong S, Luo E, Gu M, Shen X, Jiang Y, Liu Y, Liu H. Baicalin attenuates high fat diet-induced obesity and liver dysfunction: Dose-response and potential role of CaMKKbeta/AMPK/ACC pathway. Cell Physiol Biochem. 2015; 35:2349-2359.

33. Rayalam S, Yang JY, Ambati S, Della-Fera MA, Baile CA. Resveratrol induces apoptosis and inhibits adipogenesis in 3T3-L1 adipocytes. Phytother Res. 2008; 22:1367-1371.

34. Cheng CY, Yang AJ, Ekambaranellore P, Huang KC, Lin WW. Anti-obesity action of INDUS810, a natural compound from Trigonella foenum-graecum: AMPKdependent lipolysis effect in adipocytes. Obes Res Clin Pract. 2018.

(Received October 8, 2018; Revised December 29, 2018; Accepted December 31, 2018) 